\title{
Nonlinear amplification of the brillouin-rayleigh triplet caused by two-photon heating
}

\author{
V. B. Karpov*, V. V. Korobkin \\ Coherent and Nonlinear Optics Department, A.M.Prokhorov General Physics Institute, Russian Academy of Sciences, Vavilov Street 38 , \\ 119991 Moscow, Russia
}

Email address:

karpov@kapella.gpi.ru (V. B. Karpov)

To cite this article:

V. B. Karpov, V. V. Korobkin. Nonlinear Amplification of the Brillouin-Rayleigh Triplet Caused by Two-Photon Heating, Optics. Vol. 2, No. 1, 2013, pp. 7-16. doi: 10.11648/j.optics.20130201.12

\begin{abstract}
The thin structures of stimulated Brillouin scattering (SBS) and stimulated temperature scattering (STS) spectral components caused by two-photon heating are analyzed theoretically. In contrast to the linear (single-photon) case for two-photon heating a stokes SBS component exhibits the spectral shift depending on the pump intensity. Emergence of an anti-stokes SBS component is possible when the pump intensity is sufficiently high so that the positive two-photon thermal gain may compensate the negative electrostrictive gain. The spectral components of STS caused by linear or two-photon absorption (essentially different linear or two-photon STS-2) possess the same thin structures.
\end{abstract}

Keywords: Nonlinear Optics; Stimulated Brillouin Scattering (SBS); Stimulated Temperature Scattering (STS); Brillouin-Rayleigh Triplet; Two-Photon Heating; Stokes and Anti-Stokes Components; Near Ultraviolet Radiation; Excimer Lasers

\section{Introduction}

For high enough light intensity and coherence the well-known weak spontaneous Brillouin-Rayleigh triplet [1, 2] transforms into the powerful doublet of a "slightly" anti-stokes shifted line of stimulated temperature scattering (STS) caused by linear (single-photon) or two-photon absorption (linear or two-photon STS-2) and a "strongly" stokes shifted line of stimulated Brillouin scattering (SBS). Various experiments display the doublet's lines singly or grouped. For the near-ir spectral region (the pump wavelength is $\lambda_{1}=0.69 \div 1.06 \mu \mathrm{m}$ ) such a transformation has been originally observed in $[3,4]$. For the near-uv spectral region $\left(\lambda_{1}=193 \div 351 \mathrm{~nm}\right)$ such a transformation has been originally observed in [5, Fig. 2]. Indeed, in the previous near-uv studies [6 - 14] two-photon STS-2 lines have been associated mistakenly with SBS and linear STS-2 lines [5].

SBS is the unique high-efficiency converter of a coherent light wave (hereinafter called the pump wave that carries the pump intensity $I_{P}$ ) into a coherent hyperacoustical wave. Also SBS is the nonlinear-optical phenomenon providing phase conjugation (PC) of the best quality [15, 16], [5, Fig. $3]$. There are two physical mechanisms responsible for nonlinear amplification of the scattered and hyperacoustical waves during an SBS process $[1,2,4,17,18]$. The first one (hereinafter called the conventional SBS) is due to a local variation of pressure caused by the electrostrictive force [19 -22]. The second one (hereinafter called the thermal SBS) is attributed to a local variation of pressure caused by the thermal expansion. For the linear light absorption the thermal SBS (hereinafter called the linear thermal SBS) has been discussed in [23 - 25].

The purely conventional SBS was considered in [5], the thermal SBS was ignored. This is a quite typical approximation, used for instance in the study of the PC provided by SBS [15], when information gained from roughly measured spectral shifts is enough. It should be noted that too rough measurements can lead to a loss of new physics. Such a loss of the genuine SBS for the near-uv, the two-photon STS-2, and other effects [5] has happened in [6 - 14].

Following [5], the unshifted lines in the left sides of [5, Fig. 2] (relative to the pump ones in the right sides) correspond to the linear and two-photon STS-2; the shifted lines correspond to the genuine conventional SBS. The observation of the thin structures of these lines including the pump ones is restricted by a Fabry-Perot etalon based spectrum analyzer [5]. The spectral resolution of a Fabry-Perot etalon is limited by several $\mathrm{MHz}\left(\right.$ or $10^{-3} \mathrm{~cm}^{-1}$ ) [26]. To reach the higher spectral resolution methods of 
heterodyning and intensity fluctuations correlation should be used. An experimental high-resolution spectral profile of a Brillouin line exhibiting an antisymmetrical behavior is given in [27].

In this paper, a contribution of the two-photon heating to the thermal SBS (hereinafter called the two-photon thermal SBS) modifying the stokes and anti-stokes branches is considered. The thin structure of a two-photon STS-2 line first experimentally discovered in [5] was not studied theoretically and is also a subject of interest.

An effective linear absorption coefficient $\alpha_{\text {eff }}(\omega)$ has been introduced [24] for gases to describe the thermalization processes of the absorbed electromagnetic energy. In our analysis a total absorption coefficient [5]

$$
\alpha_{\Sigma}=\alpha+\left(I_{P} \gamma\right)
$$

should be used to describe the two-photon effect ( $\alpha$ is a linear absorption coefficient and $\gamma$ is a two-photon absorption coefficient).

\section{Mass (Bulk) and Serface Forces}

The theory of the coupling of light and elastic waves is based on the Lagrange equation [19, 20]. In [19] a nonlinear system was developed and a linearized system for small perturbations has been solved. The photoelastic coupling of a longitudinal acoustic wave in an isotropic medium was studied in [20], and the nonlinear system from [19] including the saturation effect has been solved.

The Lagrange equation [28] describes the mechanics of discrete bodies. It takes into account the mass (bulk) forces and ignores the surface ones. For continuous media both mass and surface forces should be incorporated [29, 30] and the Navier-Stokes equation is used instead of the Lagrange one. Indeed, any plane acoustic wave propagating in a continuous medium provides shear motion leading to the attenuation due to viscosity $\eta$ [31] (see (2) below).

\section{Material Equations}

Interaction of a light wave, characterized by a total electrical field vector $\boldsymbol{E}$, with an isotropic dielectric medium is described by the hydrodynamic equations linearized with respect to the small deviations of density $\Delta \rho$, temperature $\Delta T$, pressure $\Delta P$, and a macroscopic velocity vector $\boldsymbol{V}$ from the equilibrium values $\rho_{0}, T_{0}, P_{0}$, and $\boldsymbol{V}_{0}=0[1,2$, 4, 17, 32]:

$$
\begin{aligned}
& \rho_{0} \frac{\partial \mathrm{V}}{\partial \mathrm{t}}+\frac{\mathrm{v}^{2}}{\delta} \operatorname{grad}(\Delta \rho) \\
& +\frac{\mathrm{v}^{2} \beta \rho_{0}}{\delta} \operatorname{grad}(\Delta \mathrm{T})-\eta \nabla^{2} \mathrm{~V}= \\
& =\frac{\gamma^{\mathrm{e}}}{8 \pi} \operatorname{grad}\left(\mathrm{E}^{2}\right),
\end{aligned}
$$

$$
\begin{gathered}
\frac{\partial}{\partial t}(\Delta \rho)+\rho_{0} \operatorname{div}(\boldsymbol{V})=0 \\
\left(\rho_{0} c_{V} \frac{\partial}{\partial t}-\lambda_{T} \nabla^{2}\right)(\Delta T)-\frac{c_{V}(\delta-1)}{\beta} \frac{\partial}{\partial t}(\Delta \rho)= \\
=\frac{n c \alpha_{\Sigma}}{4 \pi}\left(\boldsymbol{E}^{2}\right)-\frac{1}{8 \pi}\left(\frac{\partial \varepsilon}{\partial T}\right)_{p}\left(T_{0} \frac{\partial}{\partial t}\left(\boldsymbol{E}^{2}\right)\right)
\end{gathered}
$$

Here $v=\sqrt{\frac{1}{\rho_{0} \beta_{S}}}$ is the speed of sound in a medium with adiabatic compressibility $\beta_{S}, c$ is the speed of light in vacuum, $\delta=\frac{c_{P}}{c_{V}}$ is a ratio of specific heats (a frequently used symbol $\gamma$ in (1) provides the logical connection with [5]), $\beta=-\frac{1}{\rho_{0}}\left(\frac{\partial \rho}{\partial T}\right)_{P}$ is a volumetric thermal expansion coefficient at constant pressure, $\gamma^{e}=\rho_{0}\left(\frac{\partial \varepsilon}{\partial \rho}\right)_{T}$ is an electrostriction coefficient, $\lambda_{T}$ is thermal conductivity, and $n$ is a refractive index.

In the Navier-Stokes equation (2) the pressure deviation is expressed as [23, 24, $33-35]$ :

$$
\Delta P=\frac{v^{2}}{\delta}(\Delta \rho)+\frac{v^{2}}{\delta} \rho_{0} \beta(\Delta T),
$$

and its gradient is moved into the left-hand side ( $\operatorname{grad} P_{0}=0$ ). The right-hand side of (2) represents the electrostrictive force [35]. The first and second right-hand side terms of the heat equation (4) represent heating due to the light absorption (both the linear and two-photon introduced by $\alpha_{\Sigma}$ ) and heating due to the electrocaloric effect $[1,36]$, respectively.

The Navier-Stokes equation (2) and the continuity equation (3) can be combined into one by eliminating the macroscopic velocity vector $\boldsymbol{V}$ :

$$
\begin{aligned}
& \left(\begin{array}{l}
-\frac{\partial^{2}}{\partial \mathrm{t}^{2}}+\frac{\mathrm{v}^{2}}{\delta} \nabla^{2} \\
+\frac{\eta}{\rho_{0}} \frac{\partial}{\partial \mathrm{t}} \nabla^{2}
\end{array}\right)(\Delta \rho)+\frac{\mathrm{v}^{2} \beta \rho_{0}}{\delta} \nabla^{2}(\Delta \mathrm{T})= \\
& =\frac{\gamma^{\mathrm{e}}}{8 \pi} \nabla^{2}\left(\mathrm{E}^{2}\right) .
\end{aligned}
$$

An ideal dielectric medium with uniform optical properties cannot scatter light. Both spontaneous (SP) and stimulated (NL) manners of the scattering arise from permittivity variation about the equilibrium value $\mathcal{E}_{0}$ :

$$
\begin{aligned}
& \Delta \varepsilon=\Delta^{S P} \varepsilon+\Delta^{N L} \mathcal{E}=\left(\frac{\partial \varepsilon}{\partial \rho}\right)_{T} \Delta \rho+\left(\frac{\partial \varepsilon}{\partial T}\right)_{\rho} \Delta T= \\
& =\left(\frac{\partial \varepsilon}{\partial \rho}\right)_{T}\left(\Delta^{S P} \rho+\Delta^{N L} \rho\right)+\left(\frac{\partial \varepsilon}{\partial T}\right)_{\rho}\left(\Delta^{S P} T+\Delta^{N L} T\right) .
\end{aligned}
$$


Usually $[1,2,17,32]$

$$
\begin{gathered}
\left|\left(\frac{\partial \varepsilon}{\partial \rho}\right)_{T} \Delta^{N L} \rho\right| \gg\left|\left(\frac{\partial \varepsilon}{\partial T}\right)_{\rho} \Delta^{N L} T\right|, \\
\left(\frac{\partial \varepsilon}{\partial T}\right)_{P} \approx-\beta\left(\rho_{0} \frac{\partial \varepsilon}{\partial \rho}\right)_{T} .
\end{gathered}
$$

The task has been provided by the material equations (4) and (5) involving independent variables $\Delta \rho$ and $\Delta T$, and as a new element a total absorption coefficient $\alpha_{\Sigma}$.

\section{SBS and STS Gain}

Physically speaking SBS and STS are nonresonant parametric phenomena [22, 37]. (Simulated Raman scattering is a resonant parametric phenomenon.)

Consider two counterpropagating linearly polarized plane electromagnetic waves, a pump wave and a backscattered wave, characterized by electrical field vectors $\boldsymbol{E}_{P}$ and $E_{s}$ :

$$
\begin{gathered}
\boldsymbol{E}_{P}=\frac{1}{2} \boldsymbol{e}\left\{E_{1}(z, t) \exp \left(i k_{1} z-i \omega_{1} t\right)+c . c .\right\}, \\
\boldsymbol{E}_{S}=\frac{1}{2} \boldsymbol{e}\left\{E_{2}(z, t) \exp \left(-i k_{2} z-i \omega_{2} t\right)+c . c .\right\} .
\end{gathered}
$$

Here, $\boldsymbol{e}$ is a common [21] unit vector (for definiteness sake $\left.\boldsymbol{e}=\boldsymbol{e}_{\boldsymbol{X}}\right) ; E_{1}(z, t)$ and $E_{2}(z, t)$ are complex amplitudes; $\omega_{1}, \omega_{2}$ and $k_{1}, k_{2}$ are temporal frequencies and wave numbers, respectively. A total electric field vector is

$$
\boldsymbol{E}=\boldsymbol{E}_{P}+\boldsymbol{E}_{S} .
$$

The linear (L) and nonlinear (NL) electrical induction vectors are $[21,22,37]$

$$
\begin{gathered}
\boldsymbol{D}^{L}(z, t)=\hat{\varepsilon}(\omega) \boldsymbol{E}(z, t), \\
\boldsymbol{D}^{N L}(z, t)=\hat{\varepsilon}^{N L}(\omega, z, t) \boldsymbol{E}(z, t),
\end{gathered}
$$

where $\hat{\varepsilon}(\omega)$ and $\hat{\varepsilon}^{N L}(\omega, z, t)$ are the Fourier transforms of the linear and nonlinear permittivity tensors $\hat{\varepsilon}\left(t_{1}\right)$ and $\hat{\varepsilon}^{N L}\left(t_{1}, t_{2}, t_{3}\right)$ [22,37]. For the isotropic medium the tensors are replaced by the scalars $[22,37]$ :

$$
\boldsymbol{D}^{L}(z, t)=\varepsilon(\omega) \boldsymbol{E}(z, t),
$$

$$
\begin{aligned}
& \boldsymbol{D}^{N L}(z, t)=\varepsilon^{N L}(\omega, z, t) \boldsymbol{E}(z, t) \cong \\
& \cong\left(\left(\frac{\partial \varepsilon}{\partial \rho}\right)_{T} \Delta^{N L} \rho(z, t)+\left(\frac{\partial \mathcal{\varepsilon}}{\partial T}\right)_{\rho} \Delta^{N L} T(z, t)\right) \boldsymbol{E}(z, t) \cong \\
& \cong\left(\frac{\partial \varepsilon}{\partial \rho}\right)_{T} \Delta^{N L} \rho(z, t) \boldsymbol{E}(z, t) .
\end{aligned}
$$

Accordingly, the linear and nonlinear polarization vectors are [21]

$$
\begin{gathered}
\boldsymbol{P}^{L}(z, t)=\frac{\varepsilon(\omega)-1}{4 \pi} \boldsymbol{E}(z, t), \\
\mathrm{P}^{\mathrm{NL}}(\mathrm{z}, \mathrm{t})=\frac{1}{4 \pi} \varepsilon^{\mathrm{NL}}(\omega, \mathrm{z}, \mathrm{t}) \mathrm{E}(\mathrm{z}, \mathrm{t}) \\
\cong \frac{1}{4 \pi}\left(\frac{\partial \varepsilon}{\partial \rho}\right)_{\mathrm{T}} \Delta^{\mathrm{NL}} \rho(\mathrm{z}, \mathrm{t}) \mathrm{E}(\mathrm{z}, \mathrm{t})
\end{gathered}
$$

The waves are coupled by the scalar electrodynamical equations $[1,2,17,32,37]$

$$
\begin{aligned}
& {\left[\nabla^{2}-\frac{\varepsilon\left(\omega_{1}\right)}{c^{2}} \frac{\partial^{2}}{\partial t^{2}}\right] \boldsymbol{E}_{P}=\frac{4 \pi}{c^{2}} \frac{\partial^{2}}{\partial t^{2}} \boldsymbol{P}_{\omega_{1}}^{N L}} \\
& {\left[\nabla^{2}-\frac{\varepsilon\left(\omega_{2}\right)}{c^{2}} \frac{\partial^{2}}{\partial t^{2}}\right] \boldsymbol{E}_{S}=\frac{4 \pi}{c^{2}} \frac{\partial^{2}}{\partial t^{2}} \boldsymbol{P}_{\omega_{2}}^{N L}}
\end{aligned}
$$

with the right-hand sides representing the nonlinear polarizations oscillating with the frequencies $\omega_{1}$ and $\omega_{2}$. For the plane waves diffraction is absent $\left(\left[\frac{\partial^{2}}{\partial x^{2}}+\frac{\partial^{2}}{\partial y^{2}}\right] \boldsymbol{E}_{P, S}(z, t)=0\right)$.

Following (4) and (5) the nonlinearity of (10) is due to the dependence of $\Delta \rho \cong \Delta^{N L} \rho$ and $\Delta T \cong \Delta^{N L} T$ on the scalar product $\boldsymbol{E}^{2}$. We seek the steady-state solution (the complex amplitudes do not depend on t) based on the slowly oscillating part of $\boldsymbol{E}^{2}$

$$
\begin{aligned}
& \left\langle E^{2}\right\rangle=\left\langle\left(E_{P}+E_{S}\right)^{2}\right\rangle=\left\langle 2 E_{p} E_{S}\right\rangle= \\
& =\frac{1}{2}\left\{E_{1}(z) E_{2}^{*}(z) \exp \left[\begin{array}{l}
-i\left(\omega_{1}-\omega_{2}\right) t \\
+i\left(k_{1}+k_{2}\right) z
\end{array}\right]+\text { c.c. }\right\},
\end{aligned}
$$

and the appropriate approximations for $\Delta^{N L} \rho$ and $\Delta^{N L} T$

$$
\begin{gathered}
\Delta^{\mathrm{NL}} \rho(z, t)=\frac{1}{2}\left\{\rho_{\mathrm{a}}(\mathrm{z}) \exp \left[\begin{array}{l}
-\mathrm{i}\left(\omega_{1}-\omega_{2}\right) \mathrm{t} \\
+\mathrm{i}\left(\mathrm{k}_{1}+\mathrm{k}_{2}\right) \mathrm{z}
\end{array}\right]+\text { c.c. }\right\}, \\
\Delta^{\mathrm{NL}} \mathrm{T}(\mathrm{z}, \mathrm{t})=\frac{1}{2}\left\{\mathrm{~T}_{\mathrm{a}}(\mathrm{z}) \exp \left[\begin{array}{l}
-\mathrm{i}\left(\omega_{1}-\omega_{2}\right) \mathrm{t} \\
+\mathrm{i}\left(\mathrm{k}_{1}+\mathrm{k}_{2}\right) \mathrm{z}
\end{array}\right]+\text { c.c. }\right\}
\end{gathered}
$$


On substitution of (6), (13) - (15) into (4) and (5), a linear system for the complex amplitudes $\rho_{a}(z), T_{a}(z)$ and for the product $E_{1}(z) E_{2}^{*}(z)$ is found

$$
\begin{aligned}
& \left(-\Omega^{2}+\frac{\mathrm{v}^{2}}{\delta} \mathrm{q}^{2}+\mathrm{i} \frac{\eta}{\rho_{0}} \mathrm{q}^{2} \Omega\right) \rho_{\mathrm{a}} \\
& +\frac{\mathrm{v}^{2} \beta \rho_{0}}{\delta} \mathrm{q}^{2} \mathrm{~T}_{\mathrm{a}}=\frac{1}{8 \pi}\left(\rho_{0} \frac{\partial \varepsilon}{\partial \rho}\right)_{\mathrm{T}} \mathrm{q}^{2} \mathrm{E}_{1} \mathrm{E}_{2}^{*} \\
& i \Omega \frac{c_{V}(\delta-1)}{\beta} \rho_{a}+\left(-i \rho_{0} c_{V} \Omega+\lambda_{T} q^{2}\right) T_{a}= \\
& =\frac{1}{4 \pi} n c \alpha_{\Sigma} E_{1} E_{2}^{*}-\frac{i}{8 \pi}\left(\rho_{0} \frac{\partial \varepsilon}{\partial \rho}\right)_{T} \beta T_{0} \Omega E_{1} E_{2}^{*},
\end{aligned}
$$

where $q=k_{1}+k_{2}, \Omega=\omega_{1}-\omega_{2}$. Solving (16) for $T_{a}$

$$
\mathrm{T}_{\mathrm{a}}=\frac{\delta}{\mathrm{q}^{2} \mathrm{v}^{2} \beta \rho_{0}}\left[\begin{array}{l}
\frac{1}{8 \pi}\left(\rho_{0} \frac{\partial \varepsilon}{\partial \rho}\right)_{\mathrm{T}} \mathrm{q}^{2} \mathrm{E}_{1} \mathrm{E}_{2}^{*}- \\
\left(-\Omega^{2}+\frac{\mathrm{v}^{2}}{\delta} \mathrm{q}^{2}+\mathrm{i} \frac{\eta}{\rho_{0}} \mathrm{q}^{2} \Omega\right) \rho_{\mathrm{a}}
\end{array}\right]
$$

and inserting the result into (17) we obtain

$$
\begin{aligned}
& \mathrm{i} \Omega \frac{\mathrm{c}_{\mathrm{v}}(\delta-1)}{\beta} \rho_{\mathrm{a}} \\
& +\frac{\delta\left(-\mathrm{i} \rho_{0} \mathrm{c}_{\mathrm{v}} \Omega+\lambda_{\mathrm{T}} \mathrm{q}^{2}\right)}{\mathrm{q}^{2} \mathrm{v}^{2} \beta \rho_{0}}\left[\begin{array}{l}
\frac{1}{8 \pi}\left(\rho_{0} \frac{\partial \varepsilon}{\partial \rho}\right)_{\mathrm{T}} \mathrm{q}^{2} \mathrm{E}_{1} \mathrm{E}_{2}^{*} \\
-\left(\begin{array}{c}
-\Omega^{2}+\frac{\mathrm{v}^{2}}{\delta} \mathrm{q}^{2} \\
+\mathrm{i} \frac{\eta}{\rho_{0}} \mathrm{q}^{2} \Omega
\end{array}\right) \rho_{\mathrm{a}}
\end{array}\right]= \\
& =\frac{1}{4 \pi}\left[n c \alpha_{\Sigma}-\frac{\mathrm{i}}{2}\left(\rho_{0} \frac{\partial \varepsilon}{\partial \rho}\right)_{\mathrm{T}} \beta \mathrm{T}_{0} \Omega\right] \mathrm{E}_{1} \mathrm{E}_{2}^{*} .
\end{aligned}
$$

Rearrangement of $\rho_{a}$ and $E_{1} E_{2}^{*}$ into the opposite sides gives

$$
\begin{gathered}
\rho_{\mathrm{a}}\left[\begin{array}{l}
\mathrm{i} \Omega \frac{\mathrm{c}_{\mathrm{V}}(\delta-1)}{\beta}- \\
\left.\frac{\delta\left(-\mathrm{i} \rho_{0} \mathrm{c}_{\mathrm{v}} \Omega+\lambda_{\mathrm{T}} \mathrm{q}^{2}\right)}{\mathrm{q}^{2} \mathrm{v}^{2} \beta \rho_{0}}\left(\begin{array}{c}
-\Omega^{2}+\frac{\mathrm{v}^{2}}{\delta} \mathrm{q}^{2} \\
+\mathrm{i} \frac{\eta}{\rho_{0}} \mathrm{q}^{2} \Omega
\end{array}\right)\right]= \\
=\mathrm{E}_{1} \mathrm{E}_{2}^{*}\left[\begin{array}{c}
\frac{\delta\left(\mathrm{i} \rho_{0} \mathrm{c}_{\mathrm{v}} \Omega-\lambda_{\mathrm{T}} \mathrm{q}^{2}\right)}{8 \pi \mathrm{q}^{2} \mathrm{v}^{2} \beta \rho_{0}}\left(\rho_{0} \frac{\partial \varepsilon}{\partial \rho}\right)_{\mathrm{T}} \mathrm{q}^{2} \\
+\frac{1}{4 \pi}\left[\mathrm{nc} \alpha_{\Sigma}-\frac{\mathrm{i}}{2}\left(\rho_{0} \frac{\partial \varepsilon}{\partial \rho}\right)_{\mathrm{T}} \beta \mathrm{T}_{0} \Omega\right.
\end{array}\right]
\end{array}\right] .
\end{gathered}
$$

Upon multiplying the both sides by $\left(-\frac{8 \pi q^{2} v^{2} \beta \rho_{0}}{\delta}\right)$ we have

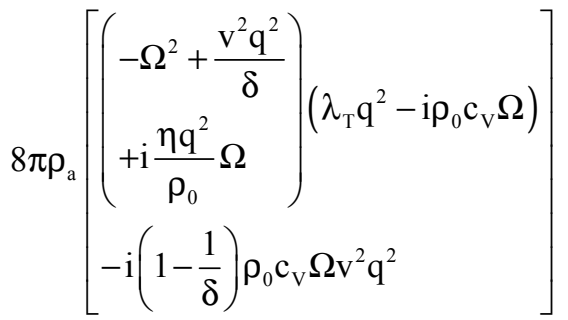

$$
\begin{aligned}
& =\mathrm{E}_{1} \mathrm{E}_{2}^{*}\left[\begin{array}{l}
-\left(i \rho_{0} \mathrm{c}_{\mathrm{v}} \Omega-\lambda_{\mathrm{T}} \mathrm{q}^{2}\right)\left(\rho_{0} \frac{\partial \varepsilon}{\partial \rho}\right)_{\mathrm{T}} \mathrm{q}^{2} \\
-\frac{2 \mathrm{q}^{2} \mathrm{v}^{2} \beta \rho_{0}}{\delta}\left[\mathrm{nc} \alpha_{\Sigma}-\frac{\mathrm{i}}{2}\left(\rho_{0} \frac{\partial \varepsilon}{\partial \rho}\right)_{\mathrm{T}} \beta \mathrm{T}_{0} \Omega\right]
\end{array}\right] \\
& =\mathrm{E}_{1} \mathrm{E}_{2}^{*}\left[\begin{array}{l}
-2 \beta \mathrm{nc} \alpha_{\Sigma} \rho_{0} \frac{\mathrm{v}^{2} \mathrm{q}^{2}}{\delta} \\
+\left(\rho_{0} \frac{\partial \varepsilon}{\partial \rho}\right)_{\mathrm{T}} \mathrm{q}^{2}\left[\begin{array}{l}
\lambda_{\mathrm{T}} \mathrm{q}^{2}-\mathrm{i} \rho_{0} \mathrm{c}_{\mathrm{V}} \Omega \\
\mathrm{i} \\
+\frac{\mathrm{i}}{\delta} \mathrm{v}^{2} \rho_{0} \beta^{2} \mathrm{~T}_{0} \Omega
\end{array}\right]
\end{array}\right] .
\end{aligned}
$$

By the use of the expressions $\beta_{S}(\delta-1)=\frac{\beta^{2} T_{0}}{\rho_{0} c_{P}}$ and $v^{2}=\frac{1}{\rho_{0} \beta_{S}}$ the other form of the last term in (18) is achieved

$$
\begin{aligned}
& \frac{\mathrm{i}}{\delta} \mathrm{v}^{2} \rho_{0} \beta^{2} \mathrm{~T}_{0} \Omega= \\
& \frac{\mathrm{i}}{\delta} \mathrm{v}^{2} \Omega \beta_{\mathrm{S}}(\delta-1) \rho_{0}^{2} \mathrm{c}_{\mathrm{P}} \\
& =\mathrm{i}\left(1-\frac{1}{\delta}\right) \rho_{0} \mathrm{c}_{\mathrm{p}} \Omega
\end{aligned}
$$

From the standpoint of a cubic nonlinear susceptibility tensor, projections of the nonlinear polarization vectors appearing in (11), (12) into the Cartesian coordinates are [37]

$$
\begin{aligned}
& \left(\mathrm{P}_{\omega_{1}, \omega_{2}}^{\mathrm{NL}}(\mathrm{z}, \mathrm{t})\right)_{\mathrm{i}} \\
& =\chi_{\mathrm{ijk} 1}^{(3)}\left(\omega_{1}, \omega_{2}, \Omega\right)(\mathrm{E}(\mathrm{z}, \mathrm{t}))_{\mathrm{j}}(\mathrm{E}(\mathrm{z}, \mathrm{t}))_{\mathrm{k}}(\mathrm{E}(\mathrm{z}, \mathrm{t}))_{1}
\end{aligned}
$$

For our case defined by (7) - (9)):

$$
\begin{aligned}
& \left(\boldsymbol{P}_{\omega_{1}, \omega_{2}}^{N L}\right)_{i}=\chi_{i x x x}^{(3)}(\boldsymbol{E})_{x}^{3}=\chi_{i x x x}^{(3)}\left(\boldsymbol{E}_{P}+\boldsymbol{E}_{S}\right)_{x}^{3}= \\
& =\chi_{i x x}^{(3)}\left[\left(\boldsymbol{E}_{P}\right)_{x}^{3}+3\left(\boldsymbol{E}_{P}\right)_{x}^{2}\left(\boldsymbol{E}_{S}\right)_{x}+3\left(\boldsymbol{E}_{P}\right)_{x}\left(\boldsymbol{E}_{S}\right)_{x}^{2}+\left(\boldsymbol{E}_{S}\right)_{x}^{3}\right]
\end{aligned}
$$

For the isotropic branch $\chi^{(3)}$ is a scalar:

$$
\begin{aligned}
& \left(\boldsymbol{P}_{\omega_{1}, \omega_{2}}^{N L}\right)_{x}=\chi^{(3)}(\boldsymbol{E})_{x}^{3}=\chi^{(3)}\left(\boldsymbol{E}_{P}+\boldsymbol{E}_{S}\right)_{x}^{3}= \\
& =\chi^{(3)}\left[\left(\boldsymbol{E}_{P}\right)_{x}^{3}+3\left(\boldsymbol{E}_{P}\right)_{x}^{2}\left(\boldsymbol{E}_{S}\right)_{x}+3\left(\boldsymbol{E}_{P}\right)_{x}\left(\boldsymbol{E}_{S}\right)_{x}^{2}+\left(\boldsymbol{E}_{S}\right)_{x}^{3}\right]
\end{aligned}
$$


On substitution of $\Delta^{N L} \rho$ from (14) into (10) in accordance with (18), (19), (20) we derive [2]:

$$
\begin{aligned}
& \chi^{(3)}=\frac{1}{4 \pi \mathrm{D}}\left(\rho_{0} \frac{\partial \varepsilon}{\partial \rho}\right)_{\mathrm{T}} \frac{1}{16 \pi \rho_{0}} \times \\
& \left\{\begin{array}{l}
\left.-2 \beta n c \alpha_{\Sigma} \rho_{0} \frac{v^{2} q^{2}}{\delta}+\left(\rho_{0} \frac{\partial \varepsilon}{\partial \rho}\right)_{\mathrm{T}} \mathrm{q}^{2}\left[\begin{array}{l}
\lambda_{\mathrm{T}} \mathrm{q}^{2} \\
-\mathrm{i} \rho_{0} \mathrm{c}_{\mathrm{V}} \Omega \\
+\mathrm{i}\left(1-\frac{1}{\delta}\right) \rho_{0} \mathrm{c}_{\mathrm{P}} \Omega
\end{array}\right]\right\} \times \\
\left\{\left[\begin{array}{l}
-\Omega^{2}+\frac{\mathrm{v}^{2} \mathrm{q}^{2}}{\delta} \\
+\mathrm{i} \frac{\eta \mathrm{q}^{2}}{\rho_{0}} \Omega
\end{array}\right]\left[\begin{array}{l}
\lambda_{\mathrm{T}} \mathrm{q}^{2} \\
-\mathrm{i} \rho_{0} \mathrm{c}_{\mathrm{V}} \Omega
\end{array}\right]-\mathrm{i}\left(1-\frac{1}{\delta}\right) \rho_{0} \mathrm{c}_{\mathrm{V}} \Omega \mathrm{v}^{2} \mathrm{q}^{2}\right.
\end{array}\right\}^{-1} .
\end{aligned}
$$

In our case $D=3$ [2]. The cubic nonlinear susceptibility (21) exhibits Rayleigh (labeled with $\mathrm{R}$ ) resonance at $|\Omega| \approx 0$ and Brillouin (labeled with $\mathrm{B}$ ) resonance at $|\Omega| \approx \Omega_{B}=q v=\left(k_{1}+k_{2}\right) v$.

Rayleigh resonances. For $\Omega \approx 0,|\Omega|<<\Omega_{B}$ (21) incorporates electrocaloric (labeled with R1) and absorptive (labeled with R2) terms:

$$
\chi^{(3) R} \approx \chi^{(3) R 1}+\chi^{(3) R 2}
$$

where

$$
\begin{gathered}
\chi^{(3) \mathrm{R} 1}=-\frac{1}{32 \pi^{2} \mathrm{D}}\left(\rho_{0} \frac{\partial \varepsilon}{\partial \rho}\right)_{\mathrm{T}}^{2} \beta_{\mathrm{S}}(\delta-1)\left[\begin{array}{c}
\frac{2-\delta}{2(\delta-1)} \\
+\frac{\mathrm{i} \Gamma_{\mathrm{R}}}{\Omega+\mathrm{i} \Gamma_{\mathrm{R}}}
\end{array}\right] \\
\chi^{(3) R 2}=\frac{1}{64 \pi^{2} D}\left(\rho_{0} \frac{\partial \varepsilon}{\partial \rho}\right)_{T} \frac{\alpha_{\Sigma} c n \beta}{\Gamma_{R} c_{P} \rho_{0}} \frac{i \Gamma_{R}}{\Omega+i \Gamma_{R}} \\
\Gamma_{R}=\frac{\lambda_{T} q^{2}}{\rho_{0} c_{P}}
\end{gathered}
$$

The imaginary parts of (22) and (23) are (as to the origin of $\beta_{R}^{e}$ and $\beta_{R}^{a}$ see (34) below)

$$
\begin{aligned}
& \operatorname{Im} \chi^{(3) \mathrm{R} 1}=-\frac{1}{32 \pi^{2} \mathrm{D}}\left(\rho_{0} \frac{\partial \varepsilon}{\partial \rho}\right)_{\mathrm{T}}^{2} \beta_{\mathrm{S}}(\delta-1) \frac{\Gamma_{\mathrm{R}} \Omega}{\Omega^{2}+\Gamma_{\mathrm{R}}^{2}} \\
& =-\frac{1}{32 \pi^{2} \mathrm{D}}\left(\rho_{0} \frac{\partial \varepsilon}{\partial \rho}\right)_{\mathrm{T}}^{2} \beta_{\mathrm{S}}(\delta-1) \frac{\Omega / \Gamma_{\mathrm{R}}}{\left(\Omega / \Gamma_{\mathrm{R}}\right)^{2}+1} \\
& =-\beta_{\mathrm{R}}^{\mathrm{e}} \frac{\Omega / \Gamma_{\mathrm{R}}}{\left(\Omega / \Gamma_{\mathrm{R}}\right)^{2}+1},
\end{aligned}
$$

$$
\begin{aligned}
& \operatorname{Im} \chi^{(3) \mathrm{R} 2}=\frac{1}{64 \pi^{2} \mathrm{D}}\left(\rho_{0} \frac{\partial \varepsilon}{\partial \rho}\right)_{\mathrm{T}} \frac{\alpha_{\Sigma} \mathrm{cn} \beta}{\Gamma_{\mathrm{R}} \mathrm{c}_{\mathrm{P}} \rho_{0}} \frac{\Gamma_{\mathrm{R}} \Omega}{\Omega^{2}+\Gamma_{\mathrm{R}}^{2}} \\
& =\frac{1}{64 \pi^{2} \mathrm{D}}\left(\rho_{0} \frac{\partial \varepsilon}{\partial \rho}\right)_{\mathrm{T}} \frac{\alpha_{\Sigma} \mathrm{cn} \beta}{\Gamma_{\mathrm{R}} \mathrm{c}_{\mathrm{P}} \rho_{0}} \frac{\Omega / \Gamma_{\mathrm{R}}}{\left(\Omega / \Gamma_{\mathrm{R}}\right)^{2}+1} \\
& =\beta_{\mathrm{R}}^{\mathrm{a}} \frac{\Omega / \Gamma_{\mathrm{R}}}{\left(\Omega / \Gamma_{\mathrm{R}}\right)^{2}+1} .
\end{aligned}
$$

Brillouin resonances. For $\Omega \approx \pm \Omega_{B}$ (21) incorporates electrostrictive (or conventional labeled with B1) and absorptive (or thermal labeled with B2) terms:

$$
\chi^{(3) B} \approx \chi^{(3) B 1}+\chi^{(3) B 2}
$$

where

$$
\begin{gathered}
\chi^{(3) \mathrm{B} 1} \frac{1}{64 \pi^{2} \mathrm{D}}\left(\rho_{0} \frac{\partial \varepsilon}{\partial \rho}\right)_{\mathrm{T}}^{2} \beta_{\mathrm{S}}(2-\delta) \frac{\rho_{0} \mathrm{v}}{\eta \mathrm{q}} \frac{\Gamma_{\mathrm{B}} / 2}{|\Omega|-\Omega_{\mathrm{B}} \pm \mathrm{i} \Gamma_{\mathrm{B}} / 2} \\
\chi^{(3) \mathrm{B} 2} \\
= \pm \frac{\mathrm{i}}{32 \pi^{2} \mathrm{D}}\left(\rho_{0} \frac{\partial \varepsilon}{\partial \rho}\right)_{\mathrm{T}} \frac{\alpha_{\Sigma} \mathrm{cn} \beta}{\Gamma_{\mathrm{B}} \mathrm{c}_{\mathrm{P}} \rho_{0}} \frac{\Gamma_{\mathrm{B}} / 2}{|\Omega|-\Omega_{\mathrm{B}} \pm \mathrm{i} \Gamma_{\mathrm{B}} / 2} \\
\Gamma_{B}=\frac{\eta q^{2}}{\rho_{0}}
\end{gathered}
$$

The bottom signs in (26), (27), (28), and (29) correspond to the stokes ( $\omega_{1}>\omega_{2}, \Omega>0,|\Omega|=\Omega$ ) and the top sings - to the anti-stokes $\left(\omega_{1}<\omega_{2}, \Omega<0,|\Omega|=-\Omega\right.$ ) spectral regions, respectively. The imaginary parts of (26) and (27) are (as to the origin of $\beta_{B}^{e}$ and $\beta_{B}^{a}$ see (34) below)

$$
\begin{aligned}
& \quad \operatorname{Im} \chi^{(3) \mathrm{B} 1} \\
& = \pm \frac{1}{64 \pi^{2} \mathrm{D}}\left(\rho_{0} \frac{\partial \varepsilon}{\partial \rho}\right)_{\mathrm{T}}^{2} \beta_{\mathrm{S}}(2-\delta) \frac{\rho_{0} \mathrm{v}}{\eta \mathrm{q}} \frac{\Gamma_{\mathrm{B}}^{2} / 4}{\left(|\Omega|-\Omega_{\mathrm{B}}\right)^{2}+\Gamma_{\mathrm{B}}^{2} / 4} \\
& = \pm \frac{1}{64 \pi^{2} \mathrm{D}}\left(\rho_{0} \frac{\partial \varepsilon}{\partial \rho}\right)_{\mathrm{T}}^{2} \beta_{\mathrm{S}}(2-\delta) \frac{\rho_{0} \mathrm{v}}{\eta \mathrm{q}} \frac{1}{\left[2\left(|\Omega|-\Omega_{\mathrm{B}}\right) / \Gamma_{\mathrm{B}}\right]^{2}+1} \\
& =\mp \beta_{\mathrm{B}}^{\mathrm{e}} \frac{1}{\left[2\left(|\Omega|-\Omega_{\mathrm{B}}\right) / \Gamma_{\mathrm{B}}\right]^{2}+1} . \\
& = \pm \frac{1}{32 \pi^{2} \mathrm{D}}\left(\rho_{0} \frac{\partial \varepsilon}{\partial \rho}\right)_{\mathrm{T}} \frac{\alpha_{\Sigma} \mathrm{cn} \beta}{\Gamma_{\mathrm{B}} \mathrm{c}_{\mathrm{P}} \rho_{0}} \frac{\left(|\Omega|-\Omega_{\mathrm{B}}\right) \Gamma_{\mathrm{B}} / 2}{\left(|\Omega|-\Omega_{\mathrm{B}}\right)^{2}+\Gamma_{\mathrm{B}}^{2} / 4} \\
& = \pm \frac{1}{32 \pi^{2} \mathrm{D}}\left(\rho_{0} \frac{\partial \varepsilon}{\partial \rho}\right)_{\mathrm{T}} \frac{\alpha_{\Sigma} \mathrm{cn} \beta}{\Gamma_{\mathrm{B}} \mathrm{c}_{\mathrm{P}} \rho_{0}} \frac{2\left(|\Omega|-\Omega_{\mathrm{B}}\right) / \Gamma_{\mathrm{B}}}{\left[2\left(|\Omega|-\Omega_{\mathrm{B}}\right) / \Gamma_{\mathrm{B}}\right]^{2}+1} \\
& =\mp \beta_{\mathrm{B}}^{\mathrm{a}} \frac{2\left(|\Omega|-\Omega_{\mathrm{B}}\right) / \Gamma_{\mathrm{B}}}{\left[2\left(|\Omega|-\Omega_{\mathrm{B}}\right) / \Gamma_{\mathrm{B}}\right]^{2}+1} .
\end{aligned}
$$

A couple of equal in magnitude to 


$$
\begin{aligned}
& \operatorname{Im} \chi_{\mathrm{MAX}}^{(3) \mathrm{B} 1}=\frac{1}{64 \pi^{2} \mathrm{D}}\left(\rho_{0} \frac{\partial \varepsilon}{\partial \rho}\right)_{\mathrm{T}}^{2} \beta_{\mathrm{S}}(2-\delta) \frac{\rho_{0} \mathrm{v}}{\eta \mathrm{q}} \\
& =\frac{\beta_{\mathrm{S}}(2-\delta) \Omega_{\mathrm{B}}}{64 \pi^{2} \mathrm{D} \Gamma_{\mathrm{B}}}\left(\rho_{0} \frac{\partial \varepsilon}{\partial \rho}\right)_{\mathrm{T}}^{2}
\end{aligned}
$$

peaks of the electrostrictive (conventional) Brillouin term defined by (28) demonstrate positive $G \propto-\operatorname{Im} \chi^{(3)}$ for $\Omega \approx \Omega_{\mathrm{B}}$, and negative $G \propto-\operatorname{Im} \chi^{(3)}$ for $\Omega \approx-\Omega_{\mathrm{B}}$ (Fig. 1a).

Four equal in magnitude to

$$
\begin{aligned}
& \operatorname{Im} \chi_{M A X}^{(3) \text { B } 2} \\
& =\frac{1}{32 \pi^{2} D}\left(\rho_{0} \frac{\partial \varepsilon}{\partial \rho}\right)_{\mathrm{T}} \frac{\alpha_{\Sigma} \operatorname{cn} \beta}{\Gamma_{\mathrm{B}} \mathrm{c}_{\mathrm{P}} \rho_{0}} \frac{\Gamma_{\mathrm{B}}^{2} / 4}{\Gamma_{\mathrm{B}}^{2} / 4+\Gamma_{\mathrm{B}}^{2} / 4} \\
& =\frac{\alpha_{\Sigma} \operatorname{cn} \beta}{64 \pi^{2} \mathrm{D} \Gamma_{\mathrm{B}} \mathrm{c}_{\mathrm{P}} \rho_{0}}\left(\rho_{0} \frac{\partial \varepsilon}{\partial \rho}\right)_{\mathrm{T}}
\end{aligned}
$$

peaks of the absorptive (thermal) Brillouin term defined by (29) demonstrate positive and negative $G \propto-\operatorname{Im} \chi^{(3)}$ both for $\Omega \approx \Omega_{\mathrm{B}}$ and for $\Omega \approx-\Omega_{\mathrm{B}}$ (Fig. 1b). The ratio of (31) and (30) provides the relative contribution of the absorptive (thermal) and electrostrictive (conventional) mechanisms into SBS gain (see Section 7):

$$
\begin{aligned}
& \frac{\operatorname{Im} \chi_{\mathrm{MAX}}^{(3) \mathrm{B} 2}}{\operatorname{Im} \chi_{\mathrm{MAX}}^{(3) \mathrm{B} 1}} \\
& =\left(\frac{\alpha_{\Sigma} \mathrm{cn} \beta}{64 \pi^{2} \mathrm{D} \Gamma_{\mathrm{B}} \mathrm{c}_{\mathrm{P}} \rho_{0}}\left(\rho_{0} \frac{\partial \varepsilon}{\partial \rho}\right)_{\mathrm{T}}\right)\left(\frac{64 \pi^{2} \mathrm{D} \Gamma_{\mathrm{B}}}{\beta_{\mathrm{S}}(2-\delta) \Omega_{\mathrm{B}}}\left(\rho_{0} \frac{\partial \varepsilon}{\partial \rho}\right)_{\mathrm{T}}^{-2}\right) \\
& =\frac{\alpha_{\Sigma} \mathrm{cn} \beta}{\mathrm{c}_{\mathrm{P}} \rho_{0} \beta_{\mathrm{S}}(2-\delta) \Omega_{\mathrm{B}}}\left(\rho_{0} \frac{\partial \varepsilon}{\partial \rho}\right)_{\mathrm{T}}^{-1} \\
& =\frac{(\delta-1)}{(2-\delta)} \frac{\alpha_{\Sigma} \mathrm{cn}}{\beta \mathrm{T}_{0} \Omega_{\mathrm{B}}}\left(\rho_{0} \frac{\partial \varepsilon}{\partial \rho}\right)_{\mathrm{T}}^{-1} \\
& =\frac{(\delta-1)}{(2-\delta)} \frac{\alpha_{\Sigma} \lambda_{1}}{4 \pi \beta \mathrm{T}_{0}} \frac{\mathrm{c} / \mathrm{v}}{\sin (\theta / 2)}\left(\rho_{0} \frac{\partial \varepsilon}{\partial \rho}\right)_{\mathrm{T}}^{-1}
\end{aligned}
$$

where

$$
\begin{gathered}
\frac{\beta}{\beta_{S}}=\frac{\rho_{0} c_{P}(\delta-1)}{\beta T_{0}}, \lambda_{1}=\frac{2 \pi c}{\omega_{1}}, k_{1}=\frac{2 \pi n}{\lambda_{1}}, \\
\Omega_{B}=q v=2 k_{1} v \sin (\theta / 2)=\frac{4 \pi v n}{\lambda_{1}} \sin (\theta / 2)
\end{gathered}
$$

$\lambda_{1}$ is the pump wavelength and $\theta$ is the angle of scattering.

On substitution of the resulting expressions for $\boldsymbol{P}^{N L}$ into (11), (12) we derive a system [17]:

$$
\left(\frac{\partial}{\partial z}+\alpha_{\Sigma}\right)\left|E_{1}(z)\right|^{2}=-G\left|E_{1}(z)\right|^{2}\left|E_{2}(z)\right|^{2},
$$

$$
\left(\frac{\partial}{\partial z}-\alpha_{\Sigma}\right)\left|E_{2}(z)\right|^{2}=-G\left|E_{1}(z)\right|^{2}\left|E_{2}(z)\right|^{2}
$$

Following (33), when the pump intensity $I_{P}(z) \propto\left|E_{1}(z)\right|^{2}$ is treated as constant over the length $L$ of nonlinear interaction, the scattered wave intensity $I_{S}(z) \propto\left|E_{2}(z)\right|^{2}$ exponentially increases along $Z$ with a gain coefficient

$$
g=G\left|E_{1}\right|^{2}-\alpha_{\Sigma}
$$

where $G \propto-\operatorname{Im} \chi^{(3)}$ is a gain parameter. The general formula for $G$ is [17] (See (24), (25), (28), and (29))

$$
\begin{aligned}
& \mathrm{G}(\Omega)= \pm \beta_{\mathrm{B}}^{\mathrm{e}} \frac{1}{1+\left(2 \Delta \Omega / \Gamma_{\mathrm{B}}\right)^{2}} \\
& \pm \beta_{\mathrm{B}}^{\mathrm{a}} \frac{2 \Delta \Omega / \Gamma_{\mathrm{B}}}{1+\left(2 \Delta \Omega / \Gamma_{\mathrm{B}}\right)^{2}} \\
& +\left(\beta_{\mathrm{R}}^{\mathrm{e}}-\beta_{\mathrm{R}}^{\mathrm{a}}\right) \frac{\Omega / \Gamma_{\mathrm{R}}}{1+\left(\Omega / \Gamma_{\mathrm{R}}\right)^{2}}
\end{aligned}
$$

where

$$
\begin{array}{r}
\Omega=\omega_{1}-\omega_{2}, \Delta \Omega=|\Omega|-\Omega_{B}, \Omega_{B}=\left(k_{1}+k_{2}\right) v, \\
\Gamma_{B}=\frac{\eta\left(k_{1}+k_{2}\right)^{2}}{\rho_{0}}, \Gamma_{R}=\frac{\lambda_{T}\left(k_{1}+k_{2}\right)^{2}}{\rho_{0} C_{P}} . \text { (35) }
\end{array}
$$

In the first two expressions of (34) signs "+" and "-" correspond to the stokes $\left(\omega_{1}>\omega_{2}, \Omega>0\right)$ and anti-stokes $\left(\omega_{1}<\omega_{2}, \Omega<0\right)$ spectral regions, respectively.

\section{SBS and ST Spectral Components for Linear Absorption}

Fig. 1 shows the spectral profiles of $G(\Omega)$ for the SBS and STS mechanisms. For linear absorption such curves can be found in $[2,18]$. A couple of the SBS curves in the vicinity of the stokes resonance can be found in [4, 17]. Excitation of an anti-stokes SBS component was not considered there.

The term proportional to $\beta_{B}^{e}$ in (34) represents the conventional SBS. The spectral profile (Fig. 1a) possesses positive stokes and negative anti-stokes values. The widths (FWHM) of resonance peaks are approximately equal to $\Gamma_{B}$.

The term proportional to $\beta_{B}^{a} \propto \alpha_{\Sigma}=\alpha$ (see (29)) represents the linear thermal SBS. The spectral profile (Fig. 1b) possesses positive and negative values in both stokes and anti-stokes regions. The widths (FWHM) of resonance 
peaks are approximately equal to $\Gamma_{B} / 2$.

The term proportional to $\beta_{R}^{a} \propto \alpha_{\Sigma}=\alpha$ (see (25)) represents the STS due to linear absorption (the linear STS-2). The spectral profile (Fig. 1b) possesses positive anti-stokes and negative stokes values. The widths (FWHM) of resonance peaks are approximately equal to $\Gamma_{R}$.

The term proportional to $\beta_{R}^{e}$ represents the STS due to an electrocaloric effect (the STS-1). The spectral profile (Fig. 1a) is mirror symmetric to that of the linear STS-2.
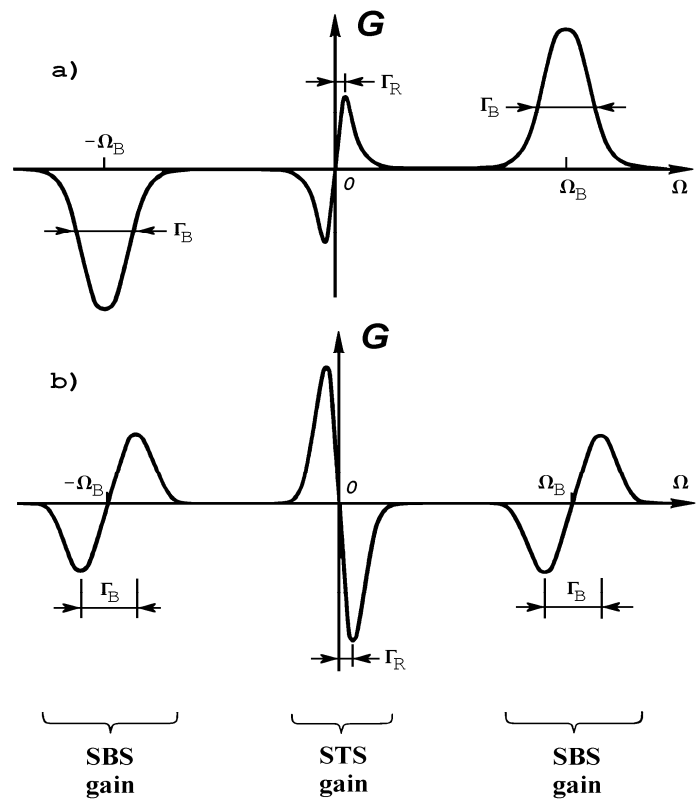

Figure 1. The gain parameter $G(\Omega)$ defined by (34) for the SBS and STS mechanisms: (a) conventional SBS and STS-1; (b) thermal SBS and STS-2.

\section{SBS and STS Spectral Components for Two-Photon Absorption}

Single-photon and two-photon transitions provide complementary spectroscopic data [17]. In analysis [5] linear (single-photon) absorption switches to two-photon one by replacing $\alpha$ with $\gamma I_{P}$. In particular, $\beta_{R}^{a}, \beta_{B}^{a} \propto \gamma I_{P}$.

Being essentially different [5], the linear STS-2 and two-photon STS-2 are characterized by the common gain curve (Fig. 1b). The shifts and widths of its resonance peaks are approximately equal to $\Gamma_{R}$, which is close to the spectral resolution of the typical experimental setup [5]. In this respect, the linear and two-photon STS-2 spectral components are experimentally indistinguishable not only from one another, but also from the STS-1 one, characterized by the mirror symmetric gain curve (Fig. 1a).

SBS contains the experimentally distinguishable [17] conventional and thermal components ( $\Omega_{B} \gg \Gamma_{B} \gg>\Gamma_{R} \approx$ spectral resolution ). In the stokes region the conventional SBS must be shifted by the thermal
SBS. For linear absorption such a shift depends on $\alpha$. For two-photon absorption such a shift depends on $\gamma_{P}$ (see Section 7). In the anti-stokes region the positive two-photon thermal values, being proportional to $\beta_{B}^{a} \propto I_{P}$, are added to the negative conventional values, being proportional to $\beta_{B}^{e}=$ const, and the positive overall SBS gain can be achieved when $I_{P}$ is sufficiently high.

\section{Overall Stokes SBS Gain}

Denoting the stokes SBS part of (34) by $\beta_{B}$ and dividing it by $\beta_{B}^{e}$, we obtain

$$
\begin{aligned}
& \left(\beta_{\mathrm{B}} / \beta_{\mathrm{B}}^{\mathrm{e}}\right)=\frac{1}{1+\left(2 \Delta \Omega / \Gamma_{\mathrm{B}}\right)^{2}} \\
& +\left(\beta_{\mathrm{B}}^{\mathrm{a}} / \beta_{\mathrm{B}}^{\mathrm{e}}\right) \frac{2 \Delta \Omega / \Gamma_{\mathrm{B}}}{1+\left(2 \Delta \Omega / \Gamma_{\mathrm{B}}\right)^{2}}
\end{aligned} .
$$

Setting

$\left(\beta_{B} / \beta_{B}^{e}\right) \equiv Z, \quad\left(\beta_{B}^{a} / \beta_{B}^{e}\right) \equiv Y, \quad\left(2 \Delta \Omega / \Gamma_{B}\right) \equiv X$, we rewrite (36) as

$$
Z(X, Y)=\frac{1}{1+X^{2}}+Y \frac{X}{1+X^{2}} .
$$

The parameters $\beta_{B}^{a}, \beta_{B}^{e}$, and $\Gamma_{B}$ are independent of $\Delta \Omega$. A variable $X$ in (37) describes the frequency shift $\Delta \Omega$. When $X=1$, the shift is $\Delta \Omega=\Gamma_{B} / 2$. A variable $Y \geq 0$ expresses a relative contribution of the thermal and conventional SBS. $Y<1$ is the realistic case of strong conventional and weak thermal mechanisms; and $Y>1$ is the unrealistic case because of the self-action and phase mismatch due to heating [5]. For linear absorption [2, 4] (see (32))

$$
Y=\frac{\beta_{B}^{a}}{\beta_{B}^{e}}=\frac{\delta-1}{2-\delta} \frac{\alpha \lambda_{1}}{4 \pi \beta T_{0}} \frac{c / v}{\sin (\theta / 2) \rho_{0}(\partial \varepsilon / \partial \rho)_{T}}
$$

where $\theta=\pi$ for the backscattering. Thus,

$$
Y=(\text { const }) \times \alpha
$$

For two-photon absorption $\alpha$ is substituted by $\chi_{P}$ in (38), and

$$
Y=(\text { const }) \times\left(\gamma I_{P}\right)
$$

According to (39) and (40), when the material properties are held constant ( $\alpha, \gamma=$ const ), a change in $I_{P}$ can cause a change in $Y$ for the two-photon thermal SBS, only. 
A function $Z(X, Y)$ can be treated as a dependence const $\times \beta_{B}(X)$ at different values of $Y$. Fig. 2 shows a three-dimensional plot of $Z=Z(X, Y)$ for $Y$ varying from 0 to 1.5 . The intersection of $Z(X, Y)$ with the plane $Y=0$ is the even function of $X$ corresponding to the conventional SBS. The contour plot in Fig. 3 demonstrates the shift more clearly.

The experimentally observed stimulated scattering spectral components are 5-times narrower [15] than the gain curves in Figs. 2 and 3. Hence, in an experiment the extra shift must be more pronounced.

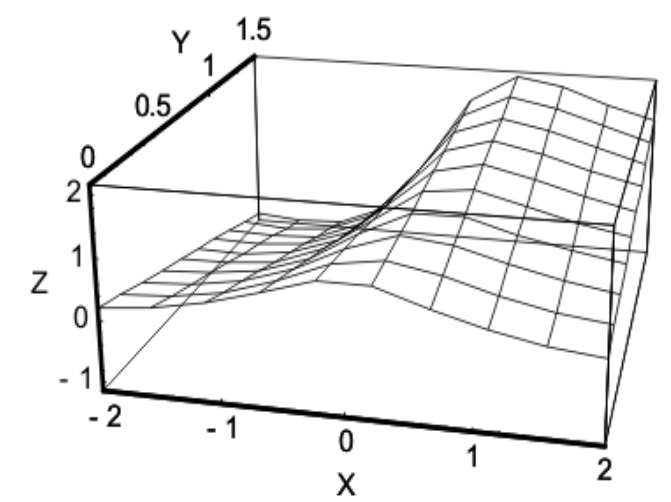

Figure 2. A three-dimensional plot of the overall stokes SBS gain parameter for the conventional and thermal mechanisms.

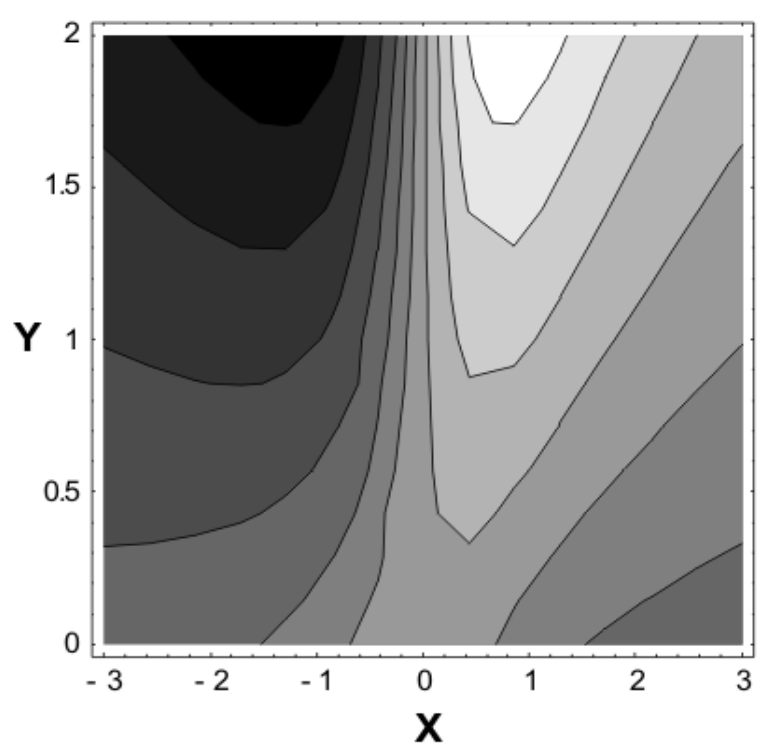

Figure 3. The contour plot corresponding to Fig. 2. The gray-scale legend is evident from Fig. 2.

\section{Numerical Estimates for Spectral Shift}

According to the relaxation theory developed by Mandelstam and Leontovich, attenuation of a hypersonic wave in a liquid is dominated by shear viscosity $[21,34,35,38]$. Therefore,

$$
\eta \approx \frac{2 \eta_{1}}{3}, k_{1}+k_{2} \approx 2 k_{1}=\frac{4 \pi n}{\lambda_{1}}
$$

where $\eta_{1}$ is a shear viscosity coefficient. From (35) we obtain

$$
\Gamma_{B}=\frac{2 \eta_{1}}{3 \rho_{0}}\left(\frac{4 \pi n}{\lambda_{1}}\right)^{2}=\frac{32 \pi^{2} n^{2} \eta_{1}}{3 \rho_{0} \lambda_{1}^{2}} .
$$

Following [5], we perform estimates for liquid hexane (C6H14), $\lambda_{1}=308 \mathrm{~nm}$, and the material parameters [1, 21, 38, 39]:

$$
\begin{aligned}
& \lambda_{1} \approx 3 \times 10^{-5} \mathrm{~cm}, \theta=\pi, \rho_{0} \approx 0.66 \frac{\mathrm{g}}{\mathrm{cm}^{3}}, \mathrm{n} \approx 1.4, \\
& \eta_{1} \approx 3.2 \times 10^{-3} \mathrm{P} \approx 3.2 \times 10^{-3} \frac{\mathrm{g}}{\mathrm{cm} \mathrm{s}}, \\
& \beta \approx 1.4 \times 10^{-3} \mathrm{~K}^{-1}, \mathrm{~T}_{0} \approx 300 \mathrm{~K}, \\
& \mathrm{v} \approx 10^{5} \frac{\mathrm{cm}}{\mathrm{s}}, \mathrm{c} \approx 3 \times 10^{10} \frac{\mathrm{cm}}{\mathrm{s}}, \rho_{0}\left(\frac{\partial \varepsilon}{\partial \rho}\right)_{\mathrm{T}} \approx 1, \delta=\frac{\mathrm{C}_{\mathrm{P}}}{\mathrm{C}_{\mathrm{V}}} \approx 1.3 .
\end{aligned}
$$

Then (41) yields

$$
\Gamma_{B} \approx 1.1 \times 10^{9} \mathrm{~Hz} \approx 0.03 \mathrm{~cm}^{-1} .
$$

Equation (38) gives

$$
Y=\frac{\beta_{B}^{a}}{\beta_{B}^{e}} \approx 0.73 \times \alpha,
$$

where $\alpha$ is measured in $\mathrm{cm}^{-1} . Y=1$ corresponds to $\alpha=1.37 \mathrm{~cm}^{-1}$.

For two-photon absorption $\alpha$ is substituted by $\mathcal{H}_{P}$ in (42), and

$$
Y=0.73 \times\left(\gamma_{P}\right) .
$$

Table 1 from [5] lists the two-photon contribution $\gamma_{P}$ obtained for the three experimental values of $I_{P}$. The maximum value is $\left(\gamma I_{P}^{\max }\right) \approx 1 \mathrm{~cm}^{-1}$ and

$$
Y^{\max }=0.73 \times\left(\chi_{P}^{\max }\right) \approx 0.73 .
$$

Due to losses and saturation the stimulated scattering is generated near the top of the curve in Fig. 2. Hence, from Fig. $3 Y \approx 0.73$ corresponds to $X \approx 0.5$, and the frequency shift is

$$
\Delta \Omega \approx \frac{\Gamma_{B}}{4} \approx 0.007 \mathrm{~cm}^{-1} .
$$

It should be noted that the SBS component was suppressed at $I_{P}^{\max }$ because of the phase mismatch associated 
with the two-photon heating [5]. For $I_{P} \approx 10^{9} \mathrm{~W} / \mathrm{cm}^{2}$ we have $\left(\gamma_{P}\right) \approx 0.1 \mathrm{~cm}^{-1}$ (see Table 1) and $Y \approx 0.07$ (see (43)). The appropriate shift (see Fig. 3) is too small, to be detected under the experimental conditions of [5]. This is not surprising because the analysis presented in [5] was focused on other issues.

Table 1. Two-photon contribution $\gamma_{I}$ to the total absorption coefficient at $\lambda_{1}=308 \mathrm{nw}$ in hexane for the three values of the pump intensity $I_{P}$

\begin{tabular}{ll}
\hline$I_{P}, W / \mathrm{cm}^{2}$ & $\gamma I_{P}, \mathrm{~cm}^{-1}$ \\
\hline $10^{10}$ & $\geq 1.0$ \\
$10^{9}$ & $\approx 0.1$ \\
$2.5 \times 10^{8}$ & $\approx 0.025$ \\
\hline
\end{tabular}

\section{Conclusions}

The basic equations describing SBS and STS are used to determine the spectral profiles of the gain. The linear (single-photon) and two-photon absorptions are compared.

In the stokes region the conventional SBS is shifted by the thermal SBS. In contrast to linear absorption, for two-photon one this shift depends on the pump intensity $I_{P}$. In the anti-stokes region the positive two-photon thermal gain being proportional to $I_{P}$ is added to the negative conventional gain, and the positive overall SBS gain can be achieved when $I_{P}$ is sufficiently high.

Estimates made for liquid hexane and the pump wavelength $308 \mathrm{~nm}$ show that the typical extra shift of the stokes SBS component is $0.007 \mathrm{~cm}^{-1}$. The spectral resolution of a Fabry-Perot etalon is limited by several $\mathrm{MHz}$ ( or $10^{-3} \mathrm{~cm}^{-1}$ ). To reach the higher spectral resolution the methods of heterodyning and intensity fluctuations correlation should be used.

For a Fabry-Perot etalon based spectrum analyzer the linear STS-2 and two-photon STS-2 components are experimentally indistinguishable not only from one another, but also from the STS-1 component.

\section{References}

[1] S.Kielich, Molecular Nonlinear Optics. Warsaw: PWN, 1977 [Moscow: Nauka, 1981].

[2] S.A.Akhmanov and N.I.Koroteev, Methods of Nonlinear Optics in Spectroscopy of Light Scattering. Moscow: Nauka, 1981 [in Russian].

[3] D.H.Rank, C.W.Cho, N.D.Foltz, and T.A.Wiggins, "Stimulated thermal Rayleigh scattering", Phys. Rev. Lett., vol. 19, pp. 828-830, 1967.

[4] V.S.Starunov and I.L.Fabelinskii, "Stimulated Mandel'shtam-Brillouin scattering and stimulated entropy (tem- perature) scattering of light", Sov. Phys. Usp., vol. 12, pp. 463-489, 1970 [Usp. Fiz. Nauk, vol. 98, pp. 441-491, 1969].

[5] V.B.Karpov and V.V.Korobkin, "Stimulated thermal scattering induced by two-photon absorption and experimental observation of genuine stimulated Brillouin scattering in the near-ultraviolet region”, Phys. Rev. A, vol. 77, p. 063812, 2008.

[6] B.J.Feldman, R.A.Fisher, A.Robert, and S.L.Shapiro, "U1traviolet phase conjugation", Optics Letters, vol. 6, No. 2, pp. $84-86,1981$.

[7] R.G.Caro and M.C.Gower, "Phase conjugation of $\mathrm{KrF}$ laser radiation", Optics Letters, vol. 6, pp. 557-559, 1981.

[8] M.C.Gower and R.G.Caro,"KrF laser with a phase-conjugate Brillouin mirror", Optics Letters, vol. 7, No. 4, pp. 162-164, 1982.

[9] M.C.Gower, "KrF laser amplifier with phase-conjugate Brillouin retroreflectors", Optics Letters, vol. 7, No. 9, pp. 423-425, 1982.

[10] E.Armandillo and D.Proch, "Highly efficient, high-quality phase-conjugate reflection at $308 \mathrm{~nm}$ using stimulated Brillouin scattering", Optics Letters, vol. 8, No. 10, pp. 523-525, 1983

[11] M.C.Gower, "Phase conjugation at 193nm", Optics Letters, vol. 8 , No. 2, pp. 70-72, 1983 .

[12] G.M.Davis and M.C.Gower, "Stimulated Brillouin scattering of a KrF laser", IEEE J. Quant. Electron., vol. 27, No. 3, pp. 496-501, 1991.

[13] S.S.Alimpiev, V.S.Bukreev, S.K.Vartapetov, I.A.Veselovskii, V.S.Nersisian, A.Z.Obidin, and A.M.Prokhorov, "Line narrowing and wavefront reversal of radiation of an $\mathrm{XeCl}$ laser", Sov. Phys.-Lebedev Inst. Reports, No. 12, pp. 12-15, 1989 [Kratk. Soobshch. Fiz., No. 12, pp. 11-13, 1989].

[14] S.S.Alimpiev, V.S.Bukreev, S.K.Vartapetov, I.A.Veselovskii, B.I.Kusakin, S.V.Lihanckii, and A.Z.Obidin, "Line narrowing and wavefront reversal of radiation of an $\mathrm{KrF}$ laser", Quant. Electron., vol. 21, pp.80-81, 1991 [Kvant. Elektron. (Moscow), vol. 18, pp. 89-90, 1991].

[15] B.Ya.Zeldovich, N.F.Pilipetsky, and V.V.Shkunov, Principles of Phase Conjugation. Berlin: Springer, 1985 [Moscow: Nauka, 1985].

[16] V.G.Dmitriev, Nonlinear Optics and Phase Conjugation. Moscow: Fizmatlit, 2003 [in Russian].

[17] Y.R.Shen, The Principles of Nonlinear Optics. New York: Wiley-Interscience, 1984 [Moscow: Nauka, 1989].

[18] B.Ya.Zel'dovich and I.I.Sobel'man," "Stimulated light scattering induced by absorption", Sov. Phys. Usp., vol. 13, pp. 307-317, 1970 [Usp. Fiz. Nauk, vol. 101, pp. 3-20, 1970].

[19] N.M.Kroll, "Excitation of hypersonic vibrations by means of photoelastic coupling of high-intensity light waves to elastic waves ”, J. Appl. Phys., vol. 36, pp. 34-44, 1965.

[20] C.L.Tang, "Saturation and spectral characteristics of the stokes emission in the stimulated Brillouin process", J. Appl. Phys., vol. 37, pp. 2945-2956, 1966.

[21] I.L.Fabelinskii, Molecular Scattering of Light. New York: 
Plenum, 1968 [Moscow: Nauka, 1965].

[22] N.Bloembergen, Nonlinear Optics. New York: W.A.Benjamin Inc., 1965 [Moscow: Mir, 1966].

[23] R.M.Herman and M.A.Gray, "Theoretical prediction of the stimulated thermal Rayleigh scattering in liquids", Phys. Rev. Lett., vol. 19, pp. 824-828, 1967.

[24] [M.A.Gray and R.M.Herman, "Nonlinear thermal Rayleigh scattering in gases", Phys. Rev., vol. 181, pp. 374-379, 1969.

[25] R.N.Enns and I.P.Batra, "Stimulated thermal scattering in the second-sound regime", Phys. Rev., vol. 180, pp. 227-232, 1969.

[26] J.M.Vaughan, "Correlation analysis and interoferometry in laser spectroscopy of scattering", in Photon Correlation and Light Beating Spectroscopy, H.Z.Cummins and E.R.Pike, Eds. New York: Plenum, 1974, pp. 432-458 [Moscow: Mir, 1978].

[27] E.R.Pike, "Theory of light scattering", in Photon Correlation and Light Beating Spectroscopy, H.Z.Cummins and E.R.Pike, Eds. New York: Plenum, 1974, pp. 17-45 [Moscow: Mir, 1978].

[28] L.D.Landau and E.M.Lifshitz, Mechanics, Course of Theoretical Physics, vol. 1. Oxford: Pergamon, 1976 [Moscow: Nauka, 1988].

[29] L.G.Loitsianskii, Mechanics of Liquids and Gases. Oxford: Pergamon, 1966 [Moscow: Drofa, 2003].

[30] L.D.Landau and E.M.Lifshitz, Theory of Elasticity, Course of Theoretical Physics, vol. 7. Oxford: Pergamon, 1986 [Moscow: Nauka, 1987].
[31] J.Lamb, "Thermal relaxation in liquids", in Physical Acoustics Principles and Methods, vol. II Part A, Properties of Gases, Liquids and Solutions, W.P.Mason, Ed. New York: Academic, 1965, pp. 222-297 [Moscow: Mir, 1968].

[32] A.Bambini, R.Vallauri, and M.Zoppi, "Nonlinear spectroscopy of Rayleigh and Mandel'stan-Brillouin scattering in liquids", in Nonlinear Spectroscopy, N.Bloembergen, Ed. Amsterdam: North-Holland Publ. Comp., 1977, pp. 569-582 [Moscow: Mir, 1979].

[33] V.E.Gusev and A.A.Karabutov, Laser Optical Acoustics. Moscow: Nauka, 1991 [in Russian].

[34] I.G.Mikhailov, V.A.Solov'ev, and Yu.P.Syrnikov, Fundamentals of Molecular Acoustics. Moscow: Nauka, 1964 [in Russian].

[35] L.D.Landau and E.M.Lifshitz, Hydrodynamics, Course of Theoretical Physics, vol. 6. Oxford: Pergamon, 1984 [Moscow: Nauka, 1986].

[36] L.D.Landau and E.M.Lifshitz, Electrodynamics of Continuous Media, Course of Theoretical Physics, vol. 8. Oxford: Pergamon, 1984 [Moscow: Nauka, 1982].

[37] S.A.Akhmanov and R.V.Khokhlov, Problems of Nonlinear Optics 1962-1963. Moscow: VINITI, 1964 [in Russian].

[38] V.F.Nozdrev, The Use of Ultrasonics in Molecular Physics. Oxford: Pergamon, 1965 [Moscow: Fizmatlit, 1958].

[39] M.I.Shakhparonov, Methods for Studying Heat Motion of Molecules and Structure of Liquids. Moscow: Moscow State University Press, 1963 [in Russian]. 